\title{
Time-dependent shot noise in multi-level quantum dot-based single-electron devices
}

\author{
V Talbo ${ }^{1}$, J Mateos $^{1}, \mathbf{S}$ Retailleau ${ }^{2}$, P Dollfus $^{2}$ and T González ${ }^{1}$ \\ ${ }^{1}$ Departamento de Física Aplicada, Universidad de Salamanca, Plaza de la Merced s/n, E-37008 \\ Salamanca, Spain \\ ${ }^{2}$ Institut d'Electronique Fondamentale, Université Paris-Sud, CNRS UMR 8622, F-91405 Orsay, France \\ E-mail: vtalbo@usal.es
}

Received 8 January 2015, revised 16 February 2015

Accepted for publication 24 February 2015

Published 31 March 2015

\begin{abstract}
By means of three-dimensional self-consistent simulation, we investigate the time- and frequency-dependent shot noise in multi-level quantum dot (QD)-based double-tunnel junction by analyzing auto-correlation functions and distribution of waiting times between consecutive tunnel events through a given barrier. We derive analytic expressions for correlation and waiting time distributions (WTDs) in the case of a maximum of two electrons in the QD. We separate the contributions of the different evolution paths of the number of electrons in the dot between two consecutive current pulses, called 'basic paths'. The close relation revealed between probabilities, WTDs and correlation functions associated to basic paths allows a good understanding of the specific dynamics in spectral densities. The analytic results show a perfect agreement with those obtained from numerical Monte-Carlo simulation.
\end{abstract}

Keywords: single-electron devices, shot noise, Monte-Carlo simulation, double-tunnel junction (Some figures may appear in colour only in the online journal)

\section{Introduction}

With the advent of solid-state mesoscopic and nanoscale systems controlled by a small amount of electrons, shot noise (SN), as a direct consequence of the discreteness of electronic charge, has been intensively studied over the last decades, providing complementary information about electronic transport to that given by the conductance measurements [16]. In the case of uncorrelated processes, the transport is Poissonian. The spectral density $S(\omega)$ related to $\mathrm{SN}$ is then often characterized at low frequency by its deviation to fully Poissonian SN $2 \mathrm{e}\langle I\rangle$, where $\langle I\rangle$ is the mean current, and the Fano factor is defined as the ratio $F=S(0) / 2 \mathrm{e}\langle I\rangle$.

Single-electron devices, and in particular single-electron transistors (SETs), are of great interest in nanotechnology. If the ability of SET to provide a fine control of current due to Coulomb blockade is known since the late 1980s [7-10], it is only recently that experimental Silicon quantum dot (QD)based SETs, likely to be integrated in silicon electronics, have shown good Coulomb-blockade oscillations at room temperature [11-15]. In the meantime, detection of single-electron events in devices has been performed experimentally, allowing measurements of $\mathrm{SN}$ and higher order correlation functions, mostly using a quantum point contact [16-32].

Most of these studies have been interpreted in the framework of the full counting statistics tool, developed by Levitov and Lesovik [33, 34], determining the probability distributions of electrons transferred during long time, giving access to all zero-frequency current-correlation functions. This theory has been extended to Coulomb blockade regime [35]. Previously, a formalism developed by Korotkov provided expressions for both static and frequency dependent SN in single-electron devices, depending on the tunnel transfer rates and the number of electrons in the QD [36]. Thanks to those tools, we are able to understand the behavior of zerofrequency SN, its suppression down to a sub-Poissonian Fano factor $F=0.5$ in a single-level QD where in and out tunnel transfer rates are equal $[37,38]$, or its enhancement up to super-Poissonian noise due to multi-levels in QDs [39], lifting of spin degeneracy [40-42] or inelastic cotunneling regime [43].

Although the calculation of finite frequency behavior of SN has been demonstrated [30, 44, 45], an alternative method has emerged recently, focusing on the time-domain. By 
studying the distribution of time-delay between two consecutive tunnel events, the electron waiting time distribution (WTD) tool provides useful information about the short-time physics in single-electron transport [46-56].

About a decade ago, a 3D simulator, called single-electron nanodevice simulation (SENS) was developed to study single-electron devices with geometry and bias as input data $[57,58]$. It relies on a 3D solver of Poisson and Schrödinger coupled equations, reaching wave-functions for each electron in the dot, then allowing the calculation of tunnel transfer rates depending on the number of electrons in the QD. Initially developed for the evaluation of $I-V$ characteristics in double-tunnel junctions (DTJs) [57], it has been extended to double-dot structures by introducing phonon contribution [59] and SETs by including the effect of a gate [60]. In a previous article [61], we used Korotkov's formula to calculate zerofrequency $\mathrm{SN}$ in a Si-QD based DTJ, explaining sub and super-Poissonian regimes through a comparison of tunnel transfer rates. The simulator also stands on a Monte-Carlo (MC) algorithm to follow the time-evolution of the number of electrons in QDs, giving access to all the time-dependent characteristics of the current, paving the way for the simulation of complex structures for which an analytic formalism could be difficult to find out.

In this paper, on the basis of tunneling rates calculated using the code SENS, we investigate the frequency-dependent $\mathrm{SN}$ in an Si-based DTJ within Korotkov formalism, limiting ourselves to a 3-state case (0-1-2 electrons in the QD). As a novelty here, we introduce the notion of basic paths, representing the different possible evolution paths of the number of electrons in the QD between two consecutive tunnel events through source oxide. The WTD, cross- and auto-correlations related to each basic path are determined. The close link between WTD and correlation functions, and the study of the contribution of each basic path give a clear and intuitive explanation of the overall frequency-dependent SN. A successful comparison between analytic results and MC simulations is also provided.

The paper is organized as follows. In section 2, the models used in the code SENS and the formalism for frequency-dependent correlations and WTD are briefly presented. The resulting zero-frequency and frequencydependent SN for different bias conditions and values of Fano factor are analyzed in section 3 in terms of basic paths and WTDs.

\section{Model}

\subsection{The code SENS}

The models used in the code SENS to simulate QD-based single-electron structures have been widely described in the case of simulation of DTJs [57, 58], double-dot structures [59] and SETs [60]. Here, we summarize the main stages of the calculation for an Si-QD based DTJ, as schematized on figure 1.

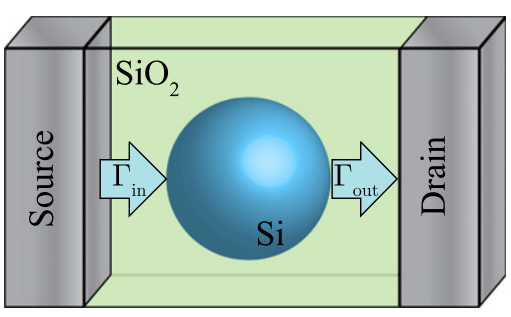

Figure 1. Schematic view of a double-tunnel junction.

The simulator relies on three stages. First, the 3D Poisson-Schrödinger coupled equations are self-consistently solved to calculate the electronic structure in the QD, depending on the bias voltage, within effective mass and Hartree approximations, proven to be correct for Si-QDs of radius greater than $1.5 \mathrm{~nm}[62,63]$. Thanks to the Hartree method, the wave-function $\psi_{i}$ and the energy level $\mu_{i}$ are provided for each electron $i$ in the dot.

Those wave functions are then used to calculate the tunneling rates between the lead $\mathrm{L}$ and the dot d containing $N$ electrons from the Fermi golden rule (weak coupling limit) as

$$
\left\{\begin{array}{l}
\Gamma_{\mathrm{Ld}}(N)=\frac{2 \pi}{\hbar}|M(N+1)|^{2} \rho_{L} l_{N} f_{L}\left(\mu_{N+1}, T_{L}\right), \\
\Gamma_{\mathrm{dL}}(N)=\frac{2 \pi}{\hbar}|M(N)|^{2} \rho_{L} g_{N}\left[1-f_{L}\left(\mu_{N}, T_{L}\right)\right],
\end{array}\right.
$$

where $g_{N}, l_{N}, T_{L}, f_{L}\left(\mu_{L}, T_{L}\right)$, and $\rho_{L}$ are the number of electrons on the energy level $\mu_{L}$, the number of free states on this level, the temperature of the lead, the Fermi function of the lead and the density of states of the lead, respectively. The tunneling matrix element $M$ is given by the Bardeen formula [58, 64].

Finally, the tunnel transfer rates are used to calculate the electrical characteristics of the device. Two methods can be used. First, from the master equation, we can deduce the static probabilities of having $N$ electrons in the $\operatorname{dot} P_{t=\infty}(N)$, and then the mean current is given by

$$
\langle I\rangle=\mathrm{e} \sum_{N} P_{t=\infty}(N)\left[\Gamma_{\mathrm{Sd}}(N)-\Gamma_{\mathrm{dS}}(N)\right] .
$$

The Fano factor, current auto-correlation function and spectral density are obtained using Korotkov formalism (equations (35), (18) and (28) in [36], respectively). The method consists mainly in the determination of the autocorrelation function by calculating the probability $P_{t_{1}, t_{2}}$ to have two tunnel events through a given junction (in this paper, the source one) at times $t_{1}$ and $t_{2}>t_{1}$, i.e.

$$
\begin{aligned}
P_{t_{1}, t_{2}}= & \Gamma_{\mathrm{Sd}}\left(N_{2}\right) \mathrm{d} t_{2} P_{t=t_{2}-t_{1}}\left(N_{2} \mid N_{1}+1\right) \\
& \times \Gamma_{\mathrm{Sd}}\left(N_{1}\right) \mathrm{d} t_{1} P_{t=\infty}\left(N_{1}\right),
\end{aligned}
$$

where $N_{1}=N\left(t_{1}\right), N_{2}=N\left(t_{2}\right)$ (before the tunnel event) and $P_{t=\tau}\left(N \mid N^{\prime}\right)$ is the time-dependent probability of having $N$ electrons in the dot at $t=\tau$ if $\mathrm{N}^{\prime}$ electrons were present at $t=0$, and is determined from the master equation. The autocorrelation function is then obtained by summing over all $N_{1}$ and $N_{2}$. The spectral density and Fano factor are deduced by a Fourier transform of the auto-correlation function. 
(a)

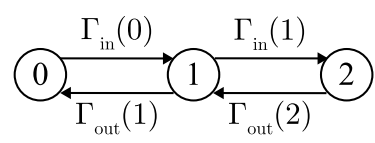

(b) (1)(1)2(12)12)(121101101)

Figure 2. Schematic description of (a) all possible transitions in a 3state case, (b) a random time-evolution of the number of electrons in the dot and (c) basic paths followed by the number of electrons in the dot between two consecutive tunnel events though source oxide. The numbers in circles are the number of electrons in the dot, in red after a tunnel event through source oxide.

Equivalently, a MC algorithm with tunnel transfer rates as input data allows us to follow the time evolution of the number of electrons in the dot, and then calculate all correlations and spectral densities.

This code is thus able to simulate and calculate static and frequency-dependent electrical characteristics in any QDbased single-electron device. However, if the behavior of single-electron currents are now well understood [57, 60], the physics of SN is more complex in multi-level QDs due to the multiple correlations between levels. This work is dedicated to the analysis of this physics.

\subsection{3-state case}

In this study, we use the values of tunnel transfer rates obtained from SENS, but for the sake of simplicity we restrict ourselves to a 3 -state case (only states with 0,1 or 2 electrons in the QD are possible), i.e. with $\Gamma_{\mathrm{Sd}}(2)=0$. We have also set the temperature to zero, so that $\Gamma_{\mathrm{dS}}=\Gamma_{\mathrm{Dd}}=0$. Hence, only four tunnel transfer rates need to be considered. In the rest of the paper, the subscript in $\Gamma_{\mathrm{Sd}}$ and $\Gamma_{\mathrm{dD}}$ will be changed to $\Gamma_{\text {in }}$ and $\Gamma_{\text {out }}$, respectively, as schematized on figure 1 .

In a 2-state case, the only time evolution of the number of electrons in the QD is $0-1-0-1-0-1-0-1 \ldots$ In the 3-state case, two events are possible if one electron is in the dot, as schematized on figure 2(a). Thus, an infinite number of timeevolution sequences is possible, for example the one illustrated in figure 2(b). Each of these time-evolution sequences are based on 4 basic paths describing the evolution of the number of electrons in the QD between two source-to-dot tunnel events. We call the basic paths (0)101 and (1)212 'pure paths' and the paths (1)2101 and (0)12 'transition paths', as they are making the transition between one pure path to an other. The number in brackets refers to the number of electrons in the dot before the first tunnel event. These four paths are schematized in figure 2(c).

Indeed, if $\Gamma_{\text {in }}(1)>\Gamma_{\text {out }}(1)$, then the (1)212 path will be the most probable to be followed when an electron has crossed the source barrier. In the contrary case, the (0)101 will be more probable. The probability of basic paths $P_{\text {path }}$ only depends on the ratio between $\Gamma_{\text {in }}(1)$ and $\Gamma_{\text {out }}(1)$ and writes

$$
\begin{aligned}
& P_{(0) 101}=\Gamma_{\text {out }}^{2}(1) /\left(\Gamma_{\text {in }}(1)+\Gamma_{\text {out }}(1)\right)^{2}, \\
& P_{(1) 212}=\Gamma_{\text {in }}^{2}(1) /\left(\Gamma_{\text {in }}(1)+\Gamma_{\text {out }}(1)\right)^{2}, \\
P_{(1) 2101}= & P_{(0) 12} \\
= & \Gamma_{\text {in }}(1) \Gamma_{\text {out }}(1) /\left(\Gamma_{\text {in }}(1)+\Gamma_{\text {out }}(1)\right)^{2} .
\end{aligned}
$$

We can also define their characteristic times $t_{\text {path }}$ as

$$
\begin{gathered}
t_{(0) 101}=\Gamma_{1}^{-1}+\Gamma_{0}^{-1}, \\
t_{(1) 212}=\Gamma_{2}^{-1}+\Gamma_{1}^{-1}, \\
t_{(0) 12}=\Gamma_{1}^{-1}, \\
t_{(1) 2101}=\Gamma_{2}^{-1}+\Gamma_{1}^{-1}+\Gamma_{0}^{-1},
\end{gathered}
$$

where $\Gamma_{0}=\Gamma_{\text {in }}(0), \Gamma_{1}=\Gamma_{\text {in }}(1)+\Gamma_{\text {out }}(1), \Gamma_{2}=\Gamma_{\text {out }}(2)$.

These characteristics times represent the mean duration of each corresponding path between two consecutive pulses, i.e. two tunneling events at the source barrier. To go further into the study of time-dependent $\mathrm{SN}$, we should consider the WTD depending on the path followed between two pulses. The WTD $\mathcal{W}(\tau)$ represents the density of probability that the time between two consecutive events is $\tau$ [46, 47]. For a Poisson process with a rate parameter $\lambda$, the WTD is $\mathcal{W}(\tau)=\lambda \mathrm{e}^{-\lambda \tau}$.

Considering that each tunnel process is Poissonian, we can calculate the WTD for each path by integrating the number of Poissonian tunnel events involved in a path ( 1 for (0)12, 2 for (1)212 and (0)101 and 3 for (1)2101):

$$
\begin{gathered}
\mathcal{W}_{(0) 12}(\tau)=\Gamma_{1} \mathrm{e}^{-\Gamma_{1} \tau}, \\
\mathcal{W}_{(0) 101}(\tau)=\frac{\Gamma_{0} \Gamma_{1}}{\Gamma_{1}-\Gamma_{0}}\left(\mathrm{e}^{-\Gamma_{0} \tau}-\mathrm{e}^{-\Gamma_{1} \tau}\right), \\
\mathcal{W}_{(1) 212}(\tau)=\frac{\Gamma_{1} \Gamma_{2}}{\Gamma_{2}-\Gamma_{1}}\left(\mathrm{e}^{-\Gamma_{1} \tau}-\mathrm{e}^{-\Gamma_{2} \tau}\right), \\
\mathcal{W}_{(1) 2101}(\tau)=\frac{\Gamma_{0} \Gamma_{1} \Gamma_{2}}{\Gamma_{1}-\Gamma_{0}} \\
\times\left[\frac{\mathrm{e}^{-\Gamma_{0} \tau}-\mathrm{e}^{-\Gamma_{2} \tau}}{\Gamma_{2}-\Gamma_{0}}-\frac{\mathrm{e}^{-\Gamma_{1} \tau}-\mathrm{e}^{-\Gamma_{2} \tau}}{\Gamma_{2}-\Gamma_{1}}\right] .
\end{gathered}
$$
WTDs

The global WTD is then deduced from probabilities and

$$
\mathcal{W}_{\text {tot }}(\tau)=\sum P_{\text {path }} \mathcal{W}_{\text {path }}(\tau)
$$

The spectral density of current, used to characterize SN, is obtained through a Fourier transform of current auto-correlation function. Each peak of current is due to either a $0 \rightarrow 1$ or a $1 \rightarrow 2$ event, and the current is decomposed in two contributions, $I_{01}$ and $I_{12}$. We define the auto- and crosscorrelation functions of those two events as

$$
\begin{gathered}
C_{01-01}(\tau)=\left\langle I_{01}(t) I_{01}(t-\tau)\right\rangle-\left\langle I_{01}\right\rangle^{2}, \\
C_{12-12}(\tau)=\left\langle I_{12}(t) I_{12}(t-\tau)\right\rangle-\left\langle I_{12}\right\rangle^{2}, \\
C_{12-01}(\tau)=\left\langle I_{01}(t) I_{12}(t-\tau)\right\rangle-\left\langle I_{01}\right\rangle\left\langle I_{12}\right\rangle,
\end{gathered}
$$




$$
C_{01-12}(\tau)=\left\langle I_{12}(t) I_{01}(t-\tau)\right\rangle-\left\langle I_{01}\right\rangle\left\langle I_{12}\right\rangle .
$$

Auto-correlations $C_{01-01}$ and $C_{12-12}$ are directly linked to the paths (0)101 and (1)212, respectively, while the crosscorrelation $C_{01-12}$ between $0 \rightarrow 1$ and subsequent $1 \rightarrow 2$ pulses are linked to (0)12 path (and the opposite for $C_{12-01}$ and the (1)2101 path). Those correlations can be determined numerically from MC simulations. Analytically, we follow the method developed by Korotkov to derive the global autocorrelation function in [36] to determine the auto- and crosscorrelations as

$$
\begin{aligned}
C_{01-01}(\tau)= & \mathrm{e}^{2} P_{t=\infty}(0) \Gamma_{\mathrm{in}}^{2}(0) P_{t=\tau}(0 \mid 1) \\
& +\mathrm{e}^{2} P_{t=\infty}(0) \Gamma_{\mathrm{in}}(0) \delta(\tau)-\left\langle I_{01}\right\rangle^{2}, \\
C_{12-12}(\tau)= & \mathrm{e}^{2} P_{t=\infty}(1) \Gamma_{\mathrm{in}}^{2}(1) P_{t=\tau}(1 \mid 2) \\
& +\mathrm{e}^{2} P_{t=\infty}(1) \Gamma_{\mathrm{in}}(1) \delta(\tau)-\left\langle I_{12}\right\rangle^{2}, \\
C_{01-12}(\tau)= & \mathrm{e}^{2} P_{t=\infty}(0) \Gamma_{\mathrm{in}}(0) P_{t=\tau}(1 \mid 1) \Gamma_{\mathrm{in}}(1) \\
& -\left\langle I_{01}\right\rangle\left\langle I_{12}\right\rangle, \\
C_{12-01}(\tau)= & \mathrm{e}^{2} P_{t=\infty}(1) \Gamma_{\mathrm{in}}(1) P_{t=\tau}(0 \mid 2) \Gamma_{\mathrm{in}}(0) \\
& -\left\langle I_{01}\right\rangle\left\langle I_{12}\right\rangle .
\end{aligned}
$$

Indeed the sum of those contributions $C_{I I}(\tau)$ leads to the general auto-correlation function of Korotkov.

The time dependences of WTD and correlation function are strongly related, because the time delay between two consecutive tunnel events through the source barrier is also the time lag on which these two events are correlated. Even though the correlation functions take into account all previous events, not only the one of the basic path considered in WTD, we expect a rather similar behavior of WTD and correlation function.

\section{Results and discussion}

The simulated DTJ consists in a $8 \mathrm{~nm}$ diameter Si QD, embedded in an $\mathrm{SiO}_{2}$ matrix, forming tunnel barriers between the dot and two metallic leads, as schematized on figure 1. The tunnel barriers on source and drain sides are $1.2 \mathrm{~nm}$ and $1.8 \mathrm{~nm}$ thick, respectively. This asymmetry has been chosen to observe both positive (PDC) and negative differential conductance (NDC) regimes on distinct Coulomb stairs. The strong influence of the bias voltage on the wave-function localization towards the drain has been shown to be the cause of NDC in DTJ [57].

\subsection{Current and $S N$}

The current and Fano factor are both plotted in figure 3(a), in the normal case, i.e. without any limitation of the number of electrons in the dot, and in the case where we restrict ourselves to three possible states. The probabilities and characteristic times of basic paths for the maximum and the minimum of zero-frequency $\mathrm{SN}$ in the 3-state case are given in table 1.

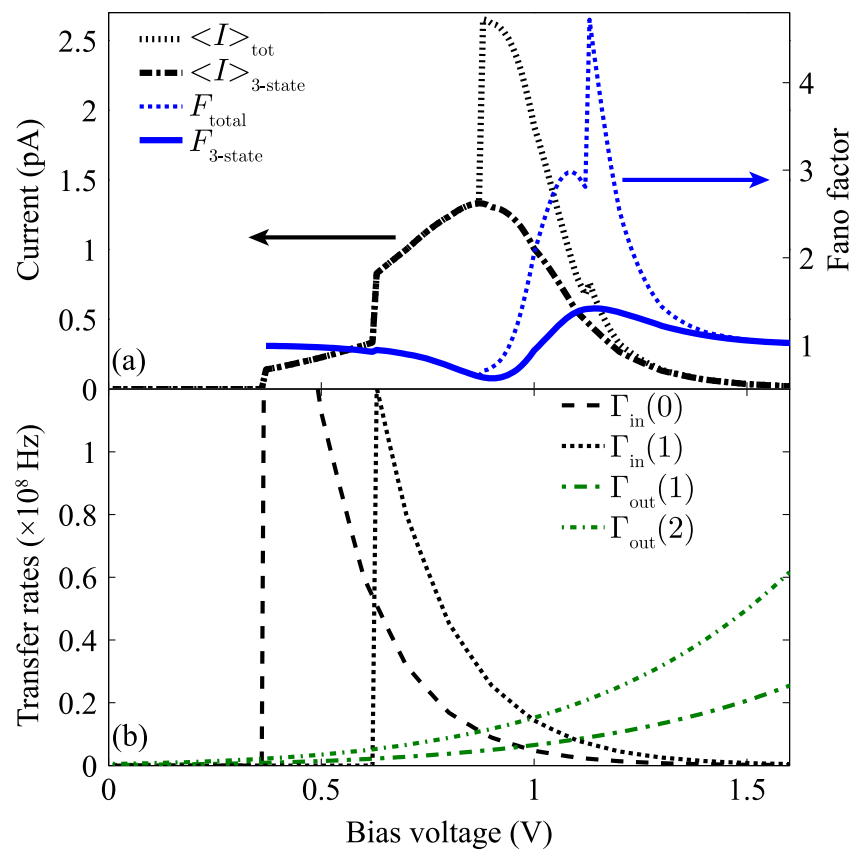

Figure 3. (a) Current and Fano factor in the normal case and the 3state case as a function of drain voltage. (b) Tunnel transfer rates involved in the 3 -state case as a function of drain voltage.

Table 1. Probabilities and characteristic times of basic paths for the extreme values of the Fano factor in the 3-state case.

\begin{tabular}{lcclcc}
\hline \multirow{2}{*}{ basic paths } & \multicolumn{2}{c}{$V=0.9 \mathrm{~V} \mathrm{~F}=0.62$} & & \multicolumn{2}{c}{$V=1.15 \mathrm{~V} \mathrm{~F}=1.42$} \\
\cline { 2 - 3 } \cline { 5 - 6 } & $P_{\text {path }}$ & $t_{\text {path }}$ & & $P_{\text {path }}$ & $t_{\text {path }}$ \\
\hline$(0) 101$ & $2 \%$ & $10^{-7} \mathrm{~s}$ & & $36 \%$ & $6 \times 10^{-7} \mathrm{~s}$ \\
$(1) 212$ & $70 \%$ & $10^{-7} \mathrm{~s}$ & & $16 \%$ & $10^{-7} \mathrm{~s}$ \\
$(0) 12$ & $14 \%$ & $3 \times 10^{-8} \mathrm{~s}$ & & $24 \%$ & $6 \times 10^{-8} \mathrm{~s}$ \\
$(1) 2101$ & $14 \%$ & $2 \times 10^{-7} \mathrm{~s}$ & & $24 \%$ & $6 \times 10^{-7} \mathrm{~s}$ \\
\hline
\end{tabular}

In the $I-V$ characteristics of the normal case, we observe two Coulomb stairs with PDC, and NDC in the following steps. In the 3-state case, the second stair shows both regimes, the NDC being a consequence of $\Gamma_{\text {out }}>\Gamma_{\text {in }}$, as seen on figure 3(b). On the first two steps of the normal case, the Fano factor remains sub-Poissonian, which is the typical behavior of a 2-state case [38]. In the 3-state case, the minimum of subPoissonian noise is $F=0.62$ at $V=0.9 \mathrm{~V}$. At this bias, $\Gamma_{\text {in }}(1)>\Gamma_{\text {out }}(1)$, promoting the (1)212 basic path up to $70 \%$, as reported in table 1 . If we were in a 2-state process (only 1 or 2 electrons in the dot), the Fano factor would be $F=0.6$. However, the transition paths (1)2101 and (0)12 occur at a rate of $14 \%$, with characteristic times twice longer and three times shorter than the (1)212 basic path, respectively. Thus, the (1)212 event that is 'lost' when a (1)2101 path occurs is almost compensated by a fast (0)12 event to come back to the pure sequence (1)212. As a consequence the SN value increases only slightly compared to the 2 -state case.

Super-Poissonian noise is reached on the third and following stairs in the normal case, due to the increasing number of states, and thus of basic paths ( 8 and 13 in the 4 and 5-state cases, respectively), and, though not shown here, to the 


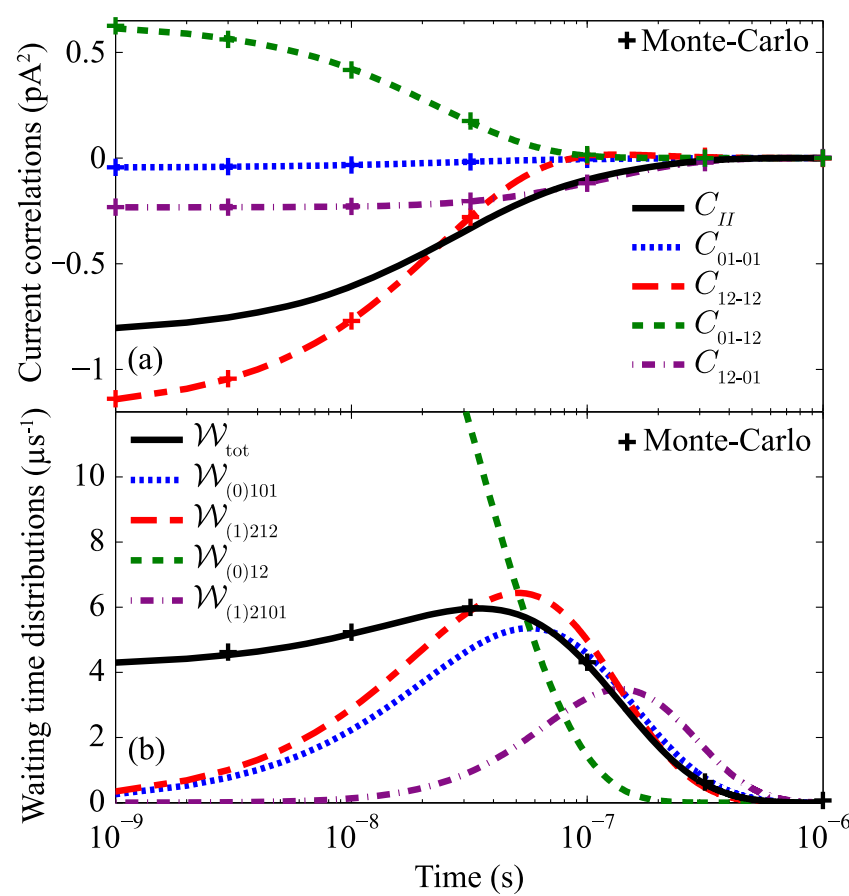

Figure 4. (a) Auto- and cross-correlations and (b) waiting time distributions corresponding to basic path and of the total current as a function of time in the case of minimum of Fano factor $(V=0.9 \mathrm{~V})$ in the 3-state case. The lines correspond to analytic results, the crosses are the results obtained using a Monte-Carlo algorithm.

increase of probability of the transition paths. In the 3-state case, super-Poissonian noise appears around $\Gamma_{\text {in }}(1)=\Gamma_{\text {out }}(1)$, even though the maximum of $\mathrm{SN}$ is reached for a slightly higher bias, $F=1.42$ at $V=1.15 \mathrm{~V}$. The most probable basic path is then (0)101, but only with a $36 \%$ rate, the rate of transition paths being at 24\%, and that of the pure path (1)212 at $16 \%$. The (0)101 and (1)2101 paths are 6 times and 10 times longer than (1)212 and (0)12, respectively, inducing a bunching phenomenon, and then a super-Poissonian noise.

\subsection{Frequency-dependent $S N$}

The current auto- and cross-correlations ((16)-(19)) as well as WTDs ((11)-(15)) for three particular biases (minimum, maximum and nearly Poissonian Fano factor $F=1.04$ ) are given in figures 4-6, respectively. For clarity reason, a logarithm scale is used for time, hiding de facto the zero-time values of auto-correlation functions. Those values are always positive and correspond to the variance of current, associated to the integral of the spectral densities which are shown in figure 7 .

The basic path (0)12 involves only one Poissonian event, implying the maximum of WTD $\mathcal{W}_{(0) 12}(\tau)$ to be located at zero. $0 \rightarrow 1$ pulses and their following $1 \rightarrow 2$ pulses are then highly correlated for low time delays, and the cross-correlations $C_{01-12}$ are always positive, before vanishing when the correlations are lost, i.e. $\mathcal{W}_{(0) 12}(\tau) \rightarrow 0$.

Conversely, we have $\mathcal{W}_{(1) 212}(0)=\mathcal{W}_{(0) 101}(0)=0$ as a consequence of Coulomb blockade: one electron has to leave the dot for another to cross the source barrier. Thus, it is

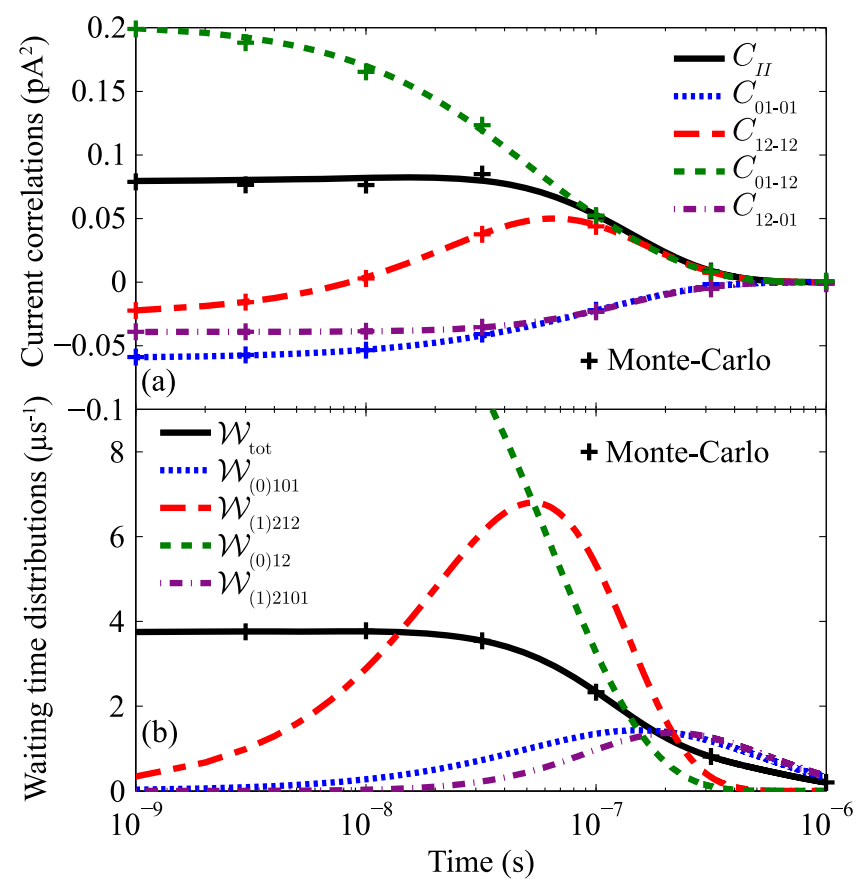

Figure 5. (a) Auto- and cross-correlations and (b) waiting time distributions corresponding to basic path and of the total current as a function of time in the case of maximum of Fano factor $(V=1.15 \mathrm{~V})$ in the 3-state case. The lines correspond to analytic results, the crosses are the results obtained using a Monte-Carlo algorithm.

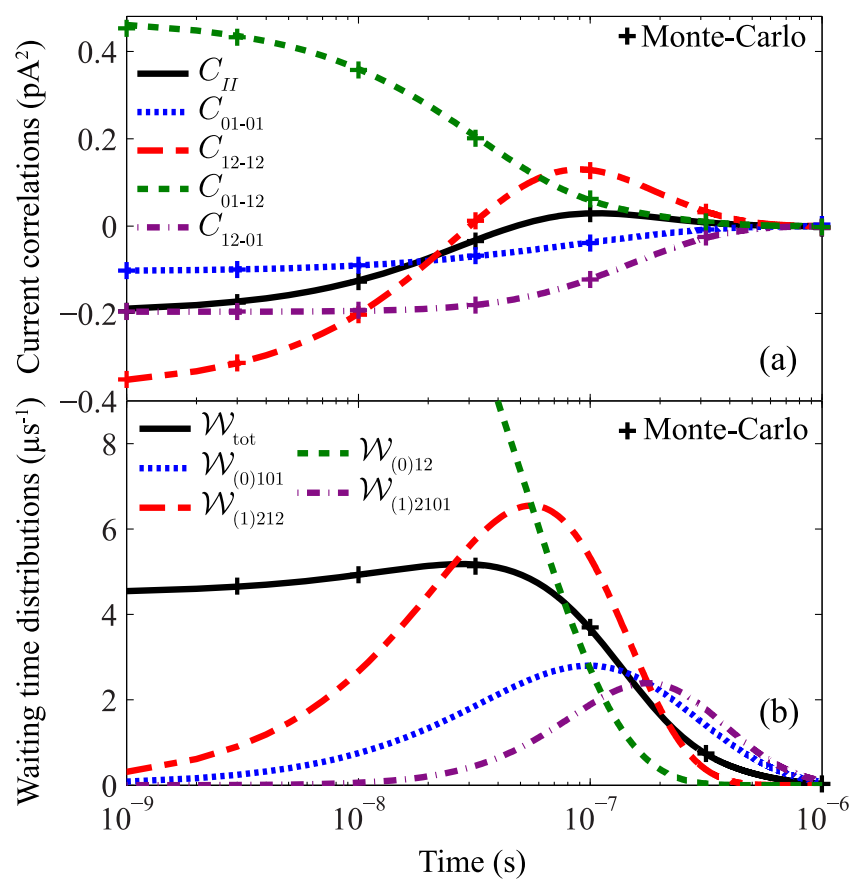

Figure 6. (a) Auto- and cross-correlations and (b) waiting time distributions corresponding to basic path and of the total current as a function of time in the case of Fano factor $F=1.04(V=1.02 \mathrm{~V})$ in the 3-state case. The lines correspond to analytic results, the crosses are the results obtained using a Monte-Carlo algorithm.

unlikely to have two consecutive $0 \rightarrow 1(1 \rightarrow 2)$ pulses with low delay time, and auto-correlations $C_{01-01}$ and $C_{12-12}$ are negative. The same behavior is observed for cross-correlations $C_{12-01}$ between $1 \rightarrow 2$ pulses and their following $0 \rightarrow 1$ 


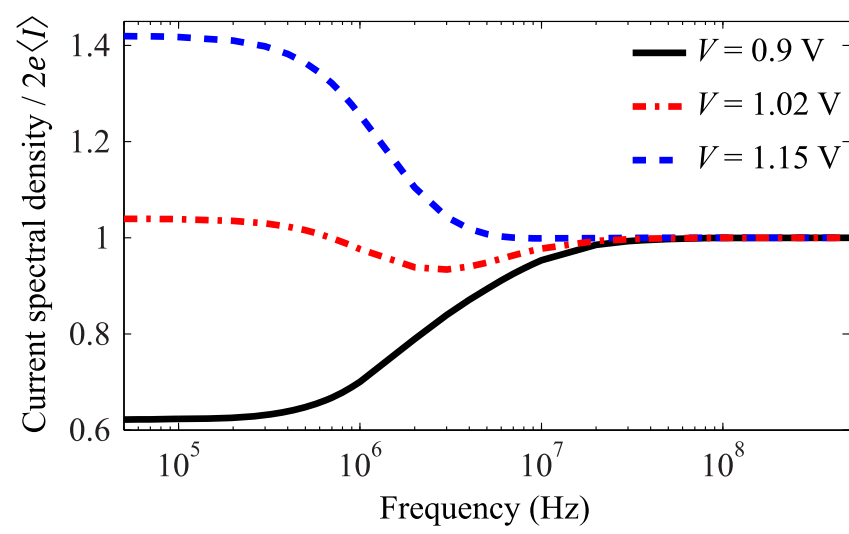

Figure 7. Current spectral density normalized by the value of a Poissonian process as a function of frequency for three different regimes.

pulses. Their absolute values at low time delays are linked with the probability of current pulses.

In the case where the minimum of Fano factor $F=0.62$ is found, plotted in figure 4, current pulses $1 \rightarrow 2$ provide $84 \%$ of the total current $(70 \%+14 \%$, see table 1$)$. This reinforces the negative contribution of $C_{12-12}$, while $C_{01-01}$ is close to zero, the value of $C_{12-01}$ being always located between those two auto-correlations. As a consequence, the global auto-correlation function $C_{I I}$ is always negative before tending to zero, typical of a sub-Poissonian Fano factor.

On the contrary, $0 \rightarrow 1$ pulses (52\%) contribute slightly more than the $1 \rightarrow 2$ ones $(48 \%)$ at the maximum of Fano factor shown in figure 5. The 'weak' negative contributions of auto-correlations $C_{01-01}$ and $C_{12-12}$, as well as $C_{12-01}$, are not sufficient to compensate the positive contribution of $C_{01-12}$, and the global auto-correlation function $C_{I I}$ is always positive, sign of a super-Poissonian zero-frequency $\mathrm{SN}$.

In all the cases, the correlations $C_{01-01}, C_{12-12}$ and $C_{12-01}$ increase in parallel with their corresponding WTD until their maximum. This increase can lead the auto-correlations $C_{12-12}$ around the maximum of $\mathcal{W}_{(1) 212}$ to go from negative to positive values, as seen on cases $F=1.15$ and $F=1.04$ in figures 5 and 6, respectively: while Coulomb blockade provides a negative contribution for $C_{12-12}$ at low delay times, the high probability to have two consecutive events during a time around which the WTD is maximum leads to a positive correlation for such values of the delay time. Remarkably, in the case of a nearly Poissonian Fano factor shown in figure 6, this behavior causes a global auto-correlation $C_{I I}$ to also switch from negative to positive values.

Finally, both WTDs and correlation functions vanish for long times. However, we have to notice that at long times the time-dependence of correlation functions and their corresponding WTDs are slightly different, as the WTDs concern only two consecutive pulses, while the correlations take into account any tunnel event with another, not only the consecutive ones. Therefore the correlation functions tend to disappear for longer times than WTDs. The path (1)2101 is the slowest of basic paths since it involves three events, hence the maximum of $\mathcal{W}_{(1) 2101}$ is located for higher time delays than $\mathcal{W}_{(1) 212}$ and $\mathcal{W}_{(0) 101}$.

The spectral densities corresponding to the previously discussed auto-correlation functions are plotted in figure 7. The spectral densities are equal to the zero-frequency value at low frequency before tending to the Poissonian value at high frequencies. In the case $V=1.02 \mathrm{~V}(F=1.04)$, studied in figure 6 , we can clearly distinguish two regimes depending on the frequency, i.e. super-Poissonian at low frequencies, and sub-Poissonian in the $\left[10^{6}-10^{7} \mathrm{~Hz}\right]$ range. This behavior is the direct consequence of the time-dependence behavior of the global auto-correlation function $C_{I I}$, explained previously: negative for low delay times and then positive for intermediate values.

Additionally, we should notice that figures 4-6 show that the MC algorithm leads to the very same results as the analytic model for the 3 -state case. This method offers the timeevolution of the number of electrons in the dot, and then of all tunnel events, based only on tunnel transfer rates, for any device. Indeed, this MC algorithm has previously shown its robustness for current calculation in different single-electron devices $[57,59,60]$. The MC method may be a good tool to understand the behavior of $\mathrm{SN}$ in case of more complex devices, hard to model analytically, such as the study of the real DTJ at finite temperature, including all basic paths depending on the number of states available, and also of double-dot structures, accounting for phonon scattering mechanisms.

\section{Conclusion}

Thanks to the ability of SENS code to provide tunnel transfer rates depending on the number of electrons in a QD, we have been able to calculate both the zero-frequency and the frequency-dependent $\mathrm{SN}$ in a given DTJ, and to separate it into different contributions, related to the basic paths followed by the system between two current pulses. The probabilities of these basic paths and the WTD statistical tool offer a clear understanding of current auto-correlation functions, and by extension of spectral densities, in both sub and super-Poissonian regimes. In particular, the remarkable behavior of a spectral density going from super to sub-Poissonian depending on the frequency is explained as a trade-off between high probability of a given basic path and high WTD for a given time range. Finally, the possibility of simulating more complex single-electron structures is open, thanks to the possibility to use a MC algorithm, instead of an analytic formalism of correlation functions.

\section{Acknowledgments}

This work has been partially supported by the Dirección General de Investigación Científica y Técnica (MINECO) through Project TEC2013-41640-R and by the Junta de Castilla y León through Project SA052U13. V Talbo wants to 
thank the European Social Fund (ESF) for financing his postdoctoral contract.

\section{References}

[1] Landauer R 1998 Nature 392 658-9

[2] Blanter Y and Büttiker M 2000 Phys. Rep. 336 1-166

[3] Blanter Y 2005 Recent Adv. Stud. Curr. Noise (preprint condmat/0511478)

[4] Esposito M, Harbola U and Mukamel S 2009 Rev. Mod. Phys. 81 1665-702

[5] Nazarov Y V 2003 Quantum Noise in Mesoscopic Physics (Berlin: Springer)

[6] Nazarov Y V and Blanter Y M 2009 Quantum Transport: Introduction to Nanoscience (Cambridge: Cambridge University Press)

[7] Averin D and Likharev K 1986 J. Low Temp. Phys. 62 345-73

[8] Likharev K 1987 IEEE Trans. Magn. 23 1142-5

[9] Likharev K 1999 Proc. IEEE 87 606-32

[10] Takahashi Y, Ono Y, Fujiwara A and Inokawa H 2002 J. Phys.: Condens. Matter 14 R995

[11] Deshpande V et al 2013 Solid-State Electron. 84 179-84

[12] Lee S, Lee Y, Song E B and Hiramoto T 2014 Nano Lett. 14 $71-7$

[13] Shin S J, Jung C S, Park B J, Yoon T K, Lee J J, Kim S J, Choi J B, Takahashi Y and Hasko D G 2010 Appl. Phys. Lett. 97103101

[14] Shin S J, Lee J J, Kang H J, Choi J B, Yang S R E, Takahashi Y and Hasko D G 2011 Nano Lett. 11 1591-7

[15] Tanahashi Y, Suzuki R, Saraya T and Hiramoto T 2014 Japan. J. Appl. Phys. 53 04EJ08

[16] Bomze Y, Gershon G, Shovkun D, Levitov L S and Reznikov M 2005 Phys. Rev. Lett. 95176601

[17] Bylander J, Duty T and Delsing P 2005 Nature 434 361-4

[18] Choi T, Ihn T, Schön S and Ensslin K 2012 Appl. Phys. Lett. 100072110

[19] Flindt C, Fricke C, Hohls F, Novotný T, Netočný K, Brandes T and Haug R J 2009 Proc. Natl Acad. Sci. USA 106 10116-9

[20] Fricke C, Hohls F, Wegscheider W and Haug R J 2007 Phys. Rev. B 76155307

[21] Fujisawa T, Hayashi T, Tomita R and Hirayama Y 2006 Science 312 1634-6

[22] Gershon G, Bomze Y, Sukhorukov E V and Reznikov M 2008 Phys. Rev. Lett. 101016803

[23] Gustavsson S, Leturcq R, Simovič B, Schleser R, Ihn T, Studerus P, Ensslin K, Driscoll D C and Gossard A C 2006 Phys. Rev. Lett. 96076605

[24] Gustavsson S, Leturcq R, Simovič B, Schleser R, Studerus P, Ihn T, Ensslin K, Driscoll D C and Gossard A C 2006 Phys. Rev. B 74195305

[25] Lu W, Ji Z, Pfeiffer L, West K W and Rimberg A J 2003 Nature 423 422-5

[26] Mahé A, Parmentier F D, Bocquillon E, Berroir J M, Glattli D C, Kontos T, Plaçais B, Fève G, Cavanna A and Jin Y 2010 Phys. Rev. B 82201309

[27] Maisi V F, Kambly D, Flindt C and Pekola J P 2014 Phys. Rev. Lett. 112036801
[28] Pekola J P, Saira O P, Maisi V F, Kemppinen A, Möttönen M, Pashkin Y A and Averin D V 2013 Rev. Mod. Phys. 85 1421-72

[29] Sukhorukov E V, Jordan A N, Gustavsson S, Leturcq R, Ihn T and Ensslin K 2007 Nat. Phys. 3 243-7

[30] Ubbelohde N, Fricke C, Flindt C, Hohls F and Haug R J 2012 Nat. Commun. 3612

[31] Watase N, Hashisaka M, Muraki K and Fujisawa T 2014 Japan. J. Appl. Phys. 53 04EJ01

[32] Zhang X C, Mazzeo G, Brataas A, Xiao M, Yablonovitch E and Jiang H W 2009 Phys. Rev. B 80 035321

[33] Levitov L and Lesovik G 1993 JETP Lett. 58230

[34] Levitov L S, Lee H and Lesovik G B 1996 J. Math. Phys. 37 4845-66

[35] Bagrets D A and Nazarov Y V 2003 Phys. Rev. B 67085316

[36] Korotkov A N 1994 Phys. Rev. B 49 10381-92

[37] Birk H, de Jong M J M and Schönenberger C 1995 Phys. Rev. Lett. 75 1610-3

[38] Hershfield S, Davies J H, Hyldgaard P, Stanton C J and Wilkins J W 1993 Phys. Rev. B 47 1967-79

[39] Belzig W 2005 Phys. Rev. B 71161301

[40] Cottet A and Belzig W 2004 Europhys. Lett. 66405

[41] Cottet A, Belzig W and Bruder C 2004 Phys. Rev. B 70 115315

[42] Ubbelohde N, Fricke C, Hohls F and Haug R J 2013 Phys. Rev. B 88041304

[43] Sukhorukov E V, Burkard G and Loss D 2001 Phys. Rev. B 63 125315

[44] Emary C, Marcos D, Aguado R and Brandes T 2007 Phys. Rev. B 76161404

[45] Marcos D, Emary C, Brandes T and Aguado R 2011 Phys. Rev. B 83125426

[46] Albert M, Flindt C and Büttiker M 2011 Phys. Rev. Lett. 107 086805

[47] Albert M, Haack G, Flindt C and Büttiker M 2012 Phys. Rev. Lett. 108186806

[48] Albert M and Devillard P 2014 Phys. Rev. B 90035431

[49] Brandes T 2008 Ann. Phys., Lpz. 17 477-96

[50] Haack G, Albert M and Flindt C 2014 Phys. Rev. B 90205429

[51] Rajabi L, Pöltl C and Governale M 2013 Phys. Rev. Lett. 111 067002

[52] Tang G M, Xu F and Wang J 2014 Phys. Rev. B 89205310

[53] Thomas K H and Flindt C 2014 Phys. Rev. B 89245420

[54] Thomas K H and Flindt C 2013 Phys. Rev. B 87121405

[55] Welack S, Esposito M, Harbola U and Mukamel S 2008 Phys. Rev. B 77195315

[56] Welack S, Mukamel S and Yan Y 2009 Europhys. Lett. 85 57008

[57] Sée J, Dollfus P and Galdin S 2006 IEEE Trans. Electron Devices 53 1268-73

[58] Sée J, Dollfus P, Galdin S and Hesto P 2006 J. Comput. Electron. 5 35-48

[59] Valentin A, Galdin-Retailleau S and Dollfus P $2009 \mathrm{~J}$. Appl. Phys. 1067

[60] Talbo V, Galdin-Retailleau S, Valentin A and Dollfus P 2011 IEEE Trans. Electron Devices 58 3286-93

[61] Talbo V, Querlioz D, Retailleau S and Dollfus P 2012 Fluctuation Noise Lett. 111242006

[62] Sée J, Dollfus P and Galdin S 2002 Phys. Rev. B 66193307

[63] Sée J, Dollfus P and Galdin S 2002 J. Appl. Phys. 92 3141-6

[64] Bardeen J 1961 Phys. Rev. Lett. 6 57-9 